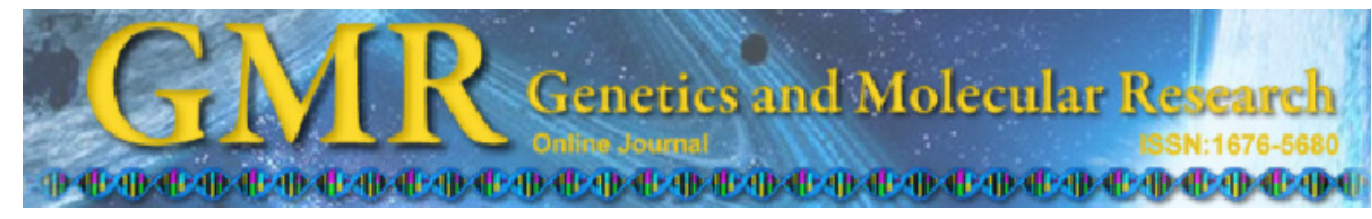

\title{
Genetic diversity of ITS sequences of Bursaphelenchus xylophilus
}

\author{
J.M.S. Cardoso, L. Fonseca and I. Abrantes \\ Department of Life Sciences, Institute of Marine Research, \\ Marine and Environmental Research Centre, University of Coimbra, \\ Coimbra, Portugal \\ Corresponding author: J.M.S. Cardoso \\ E-mail: joanasa_cardoso@hotmail.com
}

Genet. Mol. Res. 11 (4): 4508-4515 (2012)

Received January 9, 2012

Accepted June 6, 2012

Published October 15, 2012

DOI http://dx.doi.org/10.4238/2012.October.15.1

\begin{abstract}
The sequence variation of internal transcribed spacer (ITS) regions of ribosomal DNA has been routinely used for species identification and species-level phylogeny of the pinewood nematode, Bursaphelenchus xylophilus. In this study, the intraspecies ITS genetic diversity of B. xylophilus was evaluated. Three pinewood nematode isolates from the United States, Japan, and Portugal were used for polymerase chain reaction (PCR) ITS region amplification and sequencing. Multiple peaks were observed in sequencing chromatograms from ITS regions of American and Japanese isolates, suggesting the presence of more than one ribosomal sequence for each isolate. PCR products were further cloned and 10 clones of each isolate were subsequently sequenced. Additionally, the ITS regions of individual nematodes from each isolate were amplified, cloned and sequenced. Among the 3 B. xylophilus isolates analyzed, an intraspecific and intra-isolate molecular variability was found. The intra-isolate ITS molecular diversity in the American isolate was higher than that in the Japanese and Portuguese isolates. However, the level of sequence variation observed within isolates was about the same as that described among ITS repeats within individuals.
\end{abstract}

Key words: ITS diversity; Pine wilt disease; Pinewood nematode; Ribosomal DNA 


\section{INTRODUCTION}

The pinewood nematode (PWN), Bursaphelenchus xylophilus, the causal agent of pine wilt disease, is considered a native of North America. However, native American conifers are tolerant or resistant to B. xylophilus, and consequently this nematode has caused little damage (Mamiya, 1983; Jones et al., 2008). At the beginning of the 20th century, the PWN was introduced in Japan and became responsible for massive mortality of native pine trees (Pinus densiflora, P. thunbergii and P. luchuensis). The PWN then spread to China, Korea, and Taiwan (European and Mediterranean Plant Protection Organization, 2009). In Europe, the PWN was first detected in continental Portugal in the maritime pine, P. pinaster (Mota et al., 1999). It has been found more recently in Madeira Island (Fonseca et al., 2012) and Spain (Abelleira et al., 2011; Robertson et al., 2011) and represents an increasing threat to European forests.

Traditionally, the identification of PWN has been based on diagnostic morphological characters after extraction from wood samples. However, this methodology involves several time-consuming steps and requires a high level of taxonomical expertise. Additionally, PWN morphological identification can sometimes be difficult or impossible when only juvenile stages are detected or owing to the variation in the female tail of some isolates (Fonseca et al., 2008). Therefore, several molecular methods have been developed that frequently use species-specific primers by targeting several genomic regions such as satellite DNA (Castagnone et al., 2005; Cardoso et al., 2012), the heat shock protein 70 gene (Leal et al., 2005, 2007), the topoisomerase I gene (Huang et al., 2010), and internal transcribed spacer (ITS) regions of ribosomal DNA (rDNA) (Cao et al., 2005; Takeuchi et al., 2005) or by using nonspecific primers for polymerase chain reaction (PCR) amplification of the ITS regions and subsequent analysis with restriction fragment length polymorphism (RFLP) (Iwahori et al., 2000; Burgermeister et al., 2009). The ITS regions are located between the repeating array of nuclear $18 \mathrm{~S}, 5.8 \mathrm{~S}$, and $28 \mathrm{~S}$ ribosomal RNA genes and have 100-200 copies/genome. These regions are rapidly evolving and thus have been routinely used in species-level phylogeny in a wide range of organisms, including nematodes (Blouin, 2002). In B. xylophilus, the intraspecific variation of the ITS regions has been used to study the genetic structure of isolates from various regions of the world through sequencing (Zheng et al., 2003; Mota et al., 2006; Zhang et al., 2008; Nose et al., 2009) and RFLP analysis (Iwahori et al., 1998). Intraspecific diversity between virulent and avirulent $B$. xylophilus isolates has been detected using RFLP analysis with the enzyme HhaI (Aikawa et al., 2003, 2006).

ITS genetic diversity has been reported within species and individuals from a diverse range of eukaryotes, including insects (Fairley et al., 2005; Li and Wilkerson, 2007), marine sponges (Worheide et al., 2004; Vierna et al., 2010), fungi (Horton, 2002; Smith et al., 2007), and nematodes such as Meloidogyne (Hugall et al., 1999), Brugia (Areekit et al., 2009), Ascaris (Leles et al., 2010), and Pratylenchus (De Luca et al., 2011). However, to our knowledge, no data on ITS intra-isolate and intra-individual diversity in B. xylophilus or other Bursaphelenchus species have been published. In the present research, the complete nucleotide sequence of ITS rDNA regions was used to study the genetic diversity of B. xylophilus isolates from the United States, Japan, and Portugal.

\section{MATERIAL AND METHODS}

\section{Nematode isolates}

B. xylophilus isolates from the United States (BxUSA745), Japan (BxJ10), and Por- 
tugal $(\mathrm{BxPt} 12 \mathrm{G})$ were maintained in cultures of Botrytis cinerea grown on malt extract agar medium at $25^{\circ} \mathrm{C}$. Mixed developmental nematode stages were collected from the cultures, concentrated via centrifugation, and homogenized using a plastic homogenizer for DNA extraction. Three adult nematodes of each isolate were handpicked under a stereomicroscope and used individually for DNA extraction.

\section{DNA extraction and amplification of ITS regions}

Nematode DNA was extracted using a DNeasy ${ }^{\circledR}$ Blood and Tissue Mini kit (Qiagen, Germany) following manufacturer instructions. The ITS rDNA regions containing partial $18 \mathrm{~S}$ and $28 \mathrm{~S}$ and complete ITS1, 5.8S, and ITS2 sequences were amplified with PCR using 50 ng extracted DNA and 1 U Dream Taq DNA polymerase (Fermentas, USA) in 1X Dream Taq buffer, $0.2 \mathrm{mM}$ of each deoxyribonucleotide triphosphates, and $1 \mu \mathrm{M}$ primers 18 SF 5'-CGTAACAAGGTAGCTGTAG3' (Ferris et al., 1993) and 28SR 5'-TTTCACTCGCCGTTACTAAGG-3' (Vrain, 1993). All reactions were carried out in a Thermal Cycler (Bio-Rad, Spain) with an initial denaturation step of $95^{\circ} \mathrm{C}$ for $2.5 \mathrm{~min}$ followed by 40 reaction cycles of $95^{\circ} \mathrm{C}$ for $30 \mathrm{~s}$, annealing at $55^{\circ} \mathrm{C}$ for $30 \mathrm{~s}$, and extension at $72^{\circ} \mathrm{C}$ for $1 \mathrm{~min}$, and a final extension at $72^{\circ} \mathrm{C}$ for $5 \mathrm{~min}$. The resulting amplification products were purified using a QIAquick ${ }^{\circledR}$ PCR Purification Kit (Qiagen) according to manufacturer instructions, directly sequenced, and further cloned.

\section{PCR product cloning}

Purified ITS-amplified products were ligated into pGEM ${ }^{\circledR}-\mathrm{T}$ Easy Vector (Promega, USA) using $50 \mathrm{ng}$ vector in a 10- $\mu \mathrm{L}$ reaction with $3 \mathrm{U}$ T4 DNA Ligase (Promega) and $46 \mathrm{ng}$ purified PCR product in $1 \mathrm{X}$ Rapid Ligation Buffer (Promega). Ligation reactions were incubated for $1 \mathrm{~h}$ at room temperature. Then, $2 \mu \mathrm{L}$ ligation product was used to transform Escherichia coli JM109 High Efficiency Competent Cells (Promega) following manufacturer instructions.

\section{Sequencing and sequence analysis}

Plasmid DNA was extracted from E. coli cells using a QIAprep ${ }^{\circledR}$ Spin Miniprep Kit (Qiagen), and 10 selected positive clones for each B. xylophilus isolate and for each individual nematode were fully sequenced in both strands in an Automatic Sequencer 3730xl under BigDye $^{\mathrm{TM}}$ terminator cycling conditions at Macrogen Company (Korea). Sequence analysis, alignments, and determination of consensus sequences were carried out using BioEdit (Hall, 1999). Homologous sequences in the databases were searched using the Basic Local Alignment Search Tool (Altschul et al., 1997), and the beginning and end of the ITS regions were delineated based on GenBank data. Nucleotide diversity, which is the mean number of nucleotide substitutions per site \pm standard deviation, and haplotype diversity were estimated in DnaSP 5.10 (Librado and Rozas, 2009).

\section{RESULTS}

The ITS rDNA regions containing partial 18S and 28S and complete ITS1, 5.8S, and ITS2 sequences were successfully amplified for all B. xylophilus DNA samples, and 63 ITS regions were 
sequenced. Three of the regions were sequenced directly from the PCR product [GenBank accession Nos. JQ288085 (BxUSA745), JQ288086 (BxJ10), JF826227 (BxPt12G)] and 60 were cloned PCR products. The 3 ITS PCR products obtained from the mixed developmental nematode stages of BxUSA745, BxJ10, and BxPt12G were first directly sequenced. Multiple peaks in the sequencing chromatograms of BxUSA745 and BxJ10 were observed, suggesting the presence of more than one ITS ribosomal sequence for each isolate (Figure 1). From the further cloning of these PCR products, 10 clones of each of the 3 isolates were analyzed and, additionally, 10 clones for each individual specimen from each of the 3 isolates were also analyzed, within a total of 60 clones.

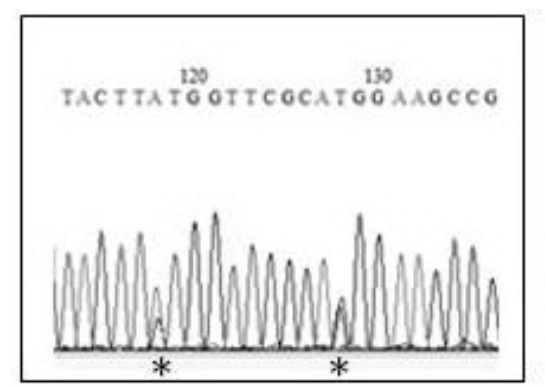

BxUSA745

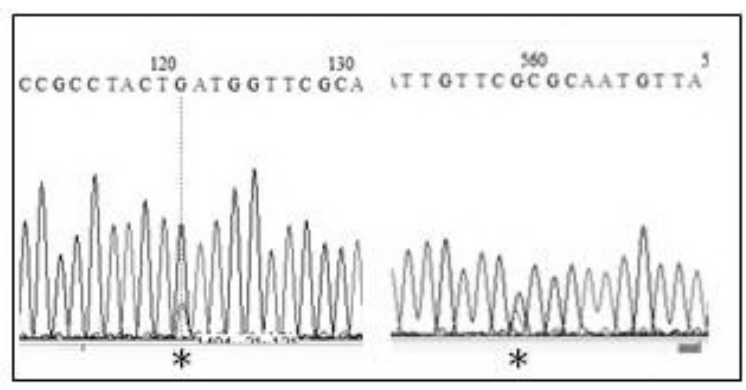

$\mathrm{BxJ} 10$

Figure 1. Sequencing chromatograms of the American (BxUSA745) and Japanese (BxJ10) Bursaphelenchus xylophilus isolate ITS regions, showing multiple peaks (asterisks).

Among the 10 mixed developmental nematode stage ITS clones of BxUSA745, sequences of 880/881 bp were identified, with 21 variable sites and 9 haplotypes. For an individual nematode of BxUSA745, sequences of $880 / 881 / 882$ bp were identified, with 15 variable sites corresponding to 9 haplotypes. From the 10 mixed developmental nematode stage ITS clones of BxJ10, sequences of $884 / 885$ bp were identified, with 10 variable sites corresponding to 8 haplotypes. For an individual nematode of BxJ10, sequences of $885 \mathrm{bp}$ were identified, with 7 variable sites and 7 haplotypes. Mixed developmental nematode stage ITS clones of BxPt12G displayed 885-bp sequences with 9 variable sites corresponding to 8 haplotypes. For an individual nematode of BxPt12G, sequences of 885 bp were identified, with 10 variable sites corresponding to 7 haplotypes (Table 1).

Overall, the ITS molecular diversity found in BxUSA745 was higher than that in BxJ10 and BxPt12G. The intra-individual ITS molecular diversities of BxUSA745 and BxJ10 were slightly lower than the intra-isolate ITS molecular diversities. Conversely, the intra-individual ITS molecular diversity of BxPt12G was slightly higher than the intra-isolate ITS molecular diversity.

The differences among ITS sequence types were due to point nucleotide substitutions and nucleotide deletions or varying numbers of repeats that resulted in different lengths of the ITS variants. The consensus sequence resulting from the alignment of all sequences was determined (Figure 2). Within the 60 clones analyzed, 45 haplotypes were found, from which 1 major haplotype is shared by 7 clones from $\mathrm{BxJ} 10$ ( 3 from mixed developmental nematode stages and 4 from individual nematodes) and 5 clones from BxPt12G ( 2 from mixed developmental nematode stages and 3 from individual nematodes). This haplotype corresponds to the one determined through the direct sequencing of BxJ10 and BxPt12G PCR products. No common haplotypes were found between BxUSA745 and the other 2 isolates. 
Table 1. Genetic diversity estimated for cloned ITS regions of American (BxUSA745), Japanese (BxJ10) and Portuguese (BxPt12G) Bursaphelenchus xylophilus isolates.

\begin{tabular}{|c|c|c|c|c|c|}
\hline Isolate & No. of clones & Cloned fragment (bp) & No. of variable sites & Haplotypes & $\mathrm{Pi} \pm$ standard deviation \\
\hline \multicolumn{6}{|l|}{ BxUSA745 } \\
\hline Mixed stages & 10 & $880 / 881$ & 21 & 9 & $0.00541 \pm 0.00061$ \\
\hline Individual & 10 & $880 / 881 / 883$ & 15 & 9 & $0.00369 \pm 0.00056$ \\
\hline Mixed stages + individual & 20 & $880 / 881 / 883$ & 33 & 18 & $0.00457 \pm 0.00047$ \\
\hline \multicolumn{6}{|l|}{ BxJ10 } \\
\hline Mixed stages & 10 & $884 / 885$ & 10 & 8 & $0.00204 \pm 0.00049$ \\
\hline Individual & 10 & 885 & 7 & 7 & $0.00158 \pm 0.00038$ \\
\hline Mixed stages + individual & 20 & $884 / 885$ & 17 & 14 & $0.00181 \pm 0.00034$ \\
\hline \multicolumn{6}{|l|}{ BxPt12G } \\
\hline Mixed stages & 10 & 885 & 9 & 8 & $0.00221 \pm 0.00047$ \\
\hline Individual & 10 & 885 & 10 & 7 & $0.00264 \pm 0.00063$ \\
\hline Mixed stages + individual & 20 & 885 & 19 & 14 & $0.00246 \pm 0.00043$ \\
\hline
\end{tabular}

1 GTGAACCTTC GGCTGGATCA TTACCGATCC TATGACACAT TTATTCGTGC TCGTCACGAT GATGCGATTG 70

71 GTGACTTCGG TTGC GCGCA TGATGGCGGT TCGATTCGCG TCGTTCCGCC TACTKRTGGT TCGCAYGGAA 140

141 GCCGAGAGGC GACCGTGCAA CGGTGAAGTC TGGGTTTCTA CGTGCTGTTG TTGAGTTGGC GTTTTACCGT 210

211 GCCGACAGAT GAGACCAGCC AGCTGCTTGC CGATTCGTTC TGGCGAGCGT AGGATTGAAA AGCCCGAGAG 280

281 GCTGCCCTGA CAAAACATTC ATTTTACATT TATTTTGTTG GAAAAGAGCT TTAAGTTACT CCGGTGGATC 350

351 ACTTGGCTCG CGGGTCGATG AAGAACGCAG TGAATTGCGA TAATAAGTAC GAATTACAGA TATTATGAGT 420

421 ACCATGTTTT TGAATGCATA TTGCGCTCTT GGGCTTTGCT CTTGAGCATA TTCGATTCAG GGTGTGTTTT 490

491 TAAACTCGAG CAGAAACGCC GACTTGTTTT TTTCAAGTTT CTGCACGTTG TGACAGTCGT CTCGCATTGT 560

561 TRCGAATG TTAGGCACCA TCTGTTTPAC GCGGTTTGTT CCGCGACCAA TATCTTCTAC GCACTGTTTG 630

631 TYCGTGCGGG GCGAGAGGGC TTCGTGCTCG ATTGTCGTGC GCGGCTAAAC CGTTTGGTGA TGTTGTTTCA 700

701 ACGGCGCGGC CGTCAGGGAC GTTCGGATGA GAATGTTTGG AGTCCTGGCT GCGGTTTGTT GAGCTTCGTC 770

771 GTGAAGCCTT GCGGGCAGTG TTGTCGGAAT TGGTTGAAAC CACCTGAGTT GGGTATGACT ACCTGCTGAA 840

841 CTTAAGCATA TCAGTAAGCA GAGGAAAAGA AACAAACATG GATTC 885

Figure 2. Consensus sequence obtained from the determined ITS sequences of the 60 Bursaphelenchus xylophilus clones. Polymorphic sites are marked with bold and indels are underlined. HhaI recognition sites are boxed [position $563 \mathrm{~A} / \mathrm{G}(\mathrm{R})$, recognition site only when G]. 18S rDNA, 5.8S rDNA and 28S rDNA surrounding ITS sequences are shaded in gray.

\section{DISCUSSION}

The complete sequence of the ITS regions from 3 isolates of $B$. xylophilus revealed intra-isolate and intra-individual genomic variability. The higher ITS genomic variability was detected in the BxUSA745 isolate, which agrees with the high diversity found among American isolates and is probably due to the ancient origin of these isolates. Interestingly, 1 of 
the 21 variable sites in the BxUSA745 mixed developmental nematode stage ITS sequences changed a recognition site for the endonuclease $H h a \mathrm{I}$, altering the predicted restriction pattern from that described for virulent B. xylophilus isolates to that of avirulent isolates (Aikawa et al., 2003). This site, nucleotide position 563 in the consensus sequence (see Figure 2), was not detected as a mutation site in the intra-individual analysis. In addition, although this site was not found as a variable site in the sequenced clones of BxJ10, it corresponds to one of the multiple peaks detected with direct PCR sequencing (see Figure 1), revealing that this isolate also contains both variants. These data indicate that the use of the ITS HhaI restriction pattern to differentiate virulent from avirulent $B$. xylophilus isolates should be reconsidered.

The major haplotype determined among the 60 clones analyzed corresponds to that determined using ITS PCR product direct sequencing of BxJ10 and BxPt12G as well as to that determined in previous studies for other Portuguese, Chinese and some Japanese isolates, which revealed no inter-isolate molecular variability in this genomic region (Mota et al., 2006; Fonseca et al., 2012). These results may reflect a unique introduction of B. xylophilus in Portugal from East Asia but not from America, which is in accordance with the conclusions of other authors using different molecular markers and techniques: inter-simple sequence repeats and RAPD-PCR (Metge and Burgermeister, 2006; Vieira et al., 2007) and sequencing of mitochondrial DNA gene regions (Moreira et al., 2011). Furthermore, the data reported here revealed that the molecular diversity found in the ITS regions of B. xylophilus isolates is mainly a result of intra-individual molecular diversity; the level of sequence variation observed among isolates is similar to that described among ITS repeats within individuals. This result is in accordance with that described for Meloidogyne species (Hugall et al., 1999) and those for other nematodes - typically $\leq 1 \%$ (Blouin, 2002). Considering that the ribosomal spacers are noncoding regions and occur in multiple copies per genome, differences in ITS sequences can be expected to occur in individual nematodes.

In conclusion, the findings of this study demonstrate that molecular diversity exists in the ITS region of B. xylophilus, revealed by intra-isolate and intra-individual ITS diversity. This diversity should be taken into account when this region is used as a molecular marker for the characterization of B. xylophilus isolates.

\section{ACKNOWLEDGMENTS}

Research supported by FEDER funds through the Programa Operacional Factores de Competitividade and by National Funds through Fundação para a Ciência e a Tecnologia (\#PTDC/AGR-CFL/098916/2008). J.M.S. Cardoso was supported by Fundação para a Ciência e a Tecnologia (post-doctoral fellowship \#SFRH/BPD/73724/2010).

\section{REFERENCES}

Abelleira A, Picoaga A, Mansilla JP and Aguin O (2011). Detection of Bursaphelenchus xylophilus, causal agent of pine wilt disease on Pinus pinaster in Northwestern Spain. Plant Dis. 95: 776.

Aikawa T, Kikuchi T and Kosaka H (2003). Demonstration of interbreeding between virulent and avirulent populations of Bursaphelenchus xylophilus (Nematoda: Aphelenchoididae) by PCR-RFLP method. Appl. Entomol. Zool. 38: 565-569.

Aikawa T, Kikuchi T and Kosaka H (2006). Population structure of Bursaphelenchus xylophilus within single Pinus thunbergii trees inoculated with two nematode isolates. Forest Pathol. 36: 1-13.

Altschul SF, Madden TL, Schaffer AA, Zhang J, et al. (1997). Gapped BLAST and PSI-BLAST: a new generation of 
protein database search programs. Nucleic Acids Res. 25: 3389-3402.

Areekit S, Singhaphan P, Khuchareontaworn S, Kanjanavas P, et al. (2009). Intraspecies variation of Brugia spp. in cat reservoirs using complete ITS sequences. Parasitol. Res. 104: 1465-1469.

Blouin MS (2002). Molecular prospecting for cryptic species of nematodes: mitochondrial DNA versus internal transcribed spacer. Int. J. Parasitol. 32: 527-531.

Burgermeister W, Braasch H, Metge K, Gu JF, et al. (2009). ITS-RFLP analysis, an efficient tool for differentiation of Bursaphelenchus species. Nematology 11: 649-668.

Cao AX, Liu XZ, Zhu SF and Lu BS (2005). Detection of the pinewood nematode, Bursaphelenchus xylophilus, using a real-time polymerase chain reaction assay. Phytopathology 95 : 566-571.

Cardoso JMS, Fonseca L and Abrantes I (2012). Direct molecular detection of the pinewood nematode, Bursaphelenchus xylophilus, from pine wood, bark and insect vector. Eur. J. Plant Pathol. 133: 419-425.

Castagnone C, Abad P and Castagnone-Sereno P (2005). Satellite DNA-based species-specific identification of single individuals of the pinewood nematode Bursaphelenchus xylophilus (Nematoda: Aphelenchoididae). Eur. J. Plant Pathol. 112: 191-193.

De Luca F, Reyes A, Troccoli A and Castillo P (2011). Molecular variability and phylogenetic relationships among different species and populations of Pratylenchus (Nematoda: Pratylenchidae) as inferred from the analysis of the ITS rDNA. Eur. J. Plant Pathol. 130: 415-426.

European and Mediterranean Plant Protection Organization (2009). Diagnostic protocols for regulated pests, Bursaphelenchus xylophilus. Bull. OEPP/EPPO 31: 61-69.

Fairley TL, Kilpatrick CW and Conn JE (2005). Intragenomic heterogeneity of internal transcribed spacer rDNA in neotropical malaria vector Anopheles aquasalis (Diptera: Culicidae). J. Med. Entomol. 42: 795-800.

Ferris VR, Ferris JM and Faghihi J (1993). Variation in spacer ribosomal DNA in some cyst-forming species of plant parasitic nematodes. Fundam. Appl. Nematol. 16: 177-184.

Fonseca L, dos Santos MCV, Santos MSNA, Curtis RHC, et al. (2008). Morpho-biometrical characterisation of Portuguese Bursaphelenchus xylophilus isolates with mucronate, digitate or round tailed females. Phytopathol. Mediterr. 47: 223-233.

Fonseca L, Cardoso JMS, Lopes A, Pestana M, et al. (2012). The pinewood nematode, Bursaphelenchus xylophilus, in Madeira Island. Helminthologia 49: 96-103.

Hall TA (1999). BioEdit: a user-friendly biological sequence alignment editor and analysis program for Windows 95/98/ NT. Nucl. Acids Symp. Ser. 41: 95-98.

Horton TR (2002). Molecular approaches to ectomycorrhizal diversity studies: variation in ITS at a local scale. Plant and Soil 244: 29-39.

Huang L, Ye JR, Wu XQ, Xu XL, et al. (2010). Detection of the pine wood nematode using a real-time PCR assay to target the DNA topoisomerase I gene. Eur. J. Plant Pathol. 127: 89-98.

Hugall A, Stanton J and Moritz C (1999). Reticulate evolution and the origins of ribosomal internal transcribed spacer diversity in apomictic Meloidogyne. Mol. Biol. Evol. 16: 157-164.

Iwahori H, Tsuda K, Kanzaki N, Izui K, et al. (1998). PCR-RFLP and sequencing analysis of ribosomal DNA of Bursaphelenchus nematodes related to pine wilt disease. Fundam. Appl. Nematol. 21: 655-666.

Iwahori H, Kanzaki N and Futai K (2000). A simple, polymerase chain reaction-restriction fragment length polymorphismaided diagnosis method for pine wilt disease. Forest Pathol. 30: 157-164.

Jones JT, Moens M, Mota M, Li H, et al. (2008). Bursaphelenchus xylophilus: opportunities in comparative genomics and molecular host-parasite interactions. Mol. Plant Pathol. 9: 357-368.

Leal I, Green M, Allen EA, Humble LM, et al. (2005). An effective PCR-based diagnostic method for the detection of Bursaphelenchus xylophilus (Nematoda: Aphelenchoididae) in wood samples from lodgepole pine. Nematology 7: 833-842.

Leal I, Green M, Allen EA, Humble LM, et al. (2007). Application of a real-time PCR method for the detection of pine wood nematode, Bursaphelenchus xylophilus, in wood samples from lodgepole pine. Nematology 9: 351-362.

Leles D, Araujo A, Vicente AC and Iniguez AM (2010). ITS1 intra-individual variability of Ascaris isolates from Brazil. Parasitol. Int. 59: 93-96.

Li C and Wilkerson RC (2007). Intragenomic rDNA ITS2 variation in the neotropical Anopheles (Nyssorhynchus) albitarsis complex (Diptera: Culicidae). J. Hered. 98: 51-59.

Librado P and Rozas J (2009). DnaSP v5: a software for comprehensive analysis of DNA polymorphism data. Bioinformatics. 25: 1451-1452.

Mamiya Y (1983). Pathology of the pine wilt disease caused by Bursaphelenchus xylophilus. Annu. Rev. Phytopathol. 21: 201-220.

Metge K and Burgermeister W (2006). Intraspecific variation in isolates of Bursaphelenchus xylophilus (Nematoda: Aphelenchoididae) revealed by ISSR and RAPD fingerprints. J. Plant Dis. Prot. 113: 275-282.

Moreira C, Pereira F, Fonseca L, Carneiro J, et al. (2011). Genetic diversity within the mitochondrial genome of 
Bursaphelenchus xylophilus. Nematropica 41: 362 .

Mota MM, Braasch H, Bravo MA, Penas AC, et al. (1999). First report of Bursaphelenchus xylophilus in Portugal and in Europe. Nematology 1: 727-734.

Mota MM, Takemoto S, Takeuchi Y, Hara N, et al. (2006). Comparative studies between Portuguese and Japanese Isolates of the pinewood nematode, Bursaphelenchus xylophilus. J. Nematol. 38: 429-433.

Nose M, Shiraishi S, Miyahara F, Ohira M, et al. (2009). Ribosomal DNA haplotype distribution of Bursaphelenchus xylophilus in Kyushu and Okinawa islands, Japan. J. Nematol. 41: 194-202.

Robertson L, Arcos SC, Escuer M, Merino RS, et al. (2011). Incidence of the pinewood nematode Bursaphelenchus xylophlius Steiner \& Buhrer, 1934 (Nickle, 1970) in Spain. Nematology 13: 755-757.

Smith ME, Douhan GW and Rizzo DM (2007). Intra-specific and intra-sporocarp ITS variation of ectomycorrhizal fungi as assessed by rDNA sequencing of sporocarps and pooled ectomycorrhizal roots from a Quercus woodland. Mycorrhiza 18: 15-22.

Takeuchi Y, Kanzaki N and Futai K (2005). A nested PCR-based method for detecting the pine wood nematode, Bursaphelenchus xylophilus, from pine wood. Nematology 7: 775-782.

Vieira P, Burgermeister W, Mota M, Metge K, et al. (2007). Lack of genetic variation of Bursaphelenchus xylophilus in Portugal revealed by RAPD-PCR analyses. J. Nematol. 39: 118-126.

Vierna J, Martinez-Lage A and Gonzalez-Tizon AM (2010). Analysis of ITS1 and ITS2 sequences in Ensis razor shells: suitability as molecular markers at the population and species levels, and evolution of these ribosomal DNA spacers. Genome 53: 23-34.

Vrain TC (1993). Restriction fragment length polymorphism separates species of the Xiphinema americanum group. $J$. Nematol. 25: 361-364.

Worheide G, Nichols SA and Goldberg J (2004). Intragenomic variation of the rDNA internal transcribed spacers in sponges (Phylum Porifera): implications for phylogenetic studies. Mol. Phylogenet. Evol. 33: 816-830.

Zhang K, Liu H, Sun J, Liu J, et al. (2008). Molecular phylogeny of geographical isolates of Bursaphelenchus xylophilus: implications on the origin and spread of this species in China and Worldwide. J. Nematol. 40: 127-137.

Zheng JW, Subbotin SA, He SS, Gu JF, et al. (2003). Molecular characterisation of some Asian isolates of Bursaphelenchus xylophilus and B. mucronatus using PCR-RFLPs and sequences of ribosomal DNA. Russ. J. Nematol. 11: 17-22. 